LA-8371

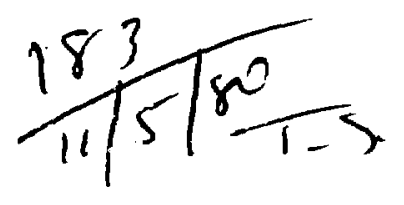

An Exact Partial Solution to the Steady-State, Compressible Fluid Flow Problems of Jet Formation and Jet Penetration

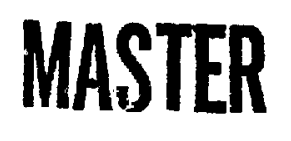

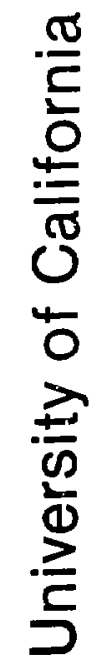




\title{
AN EXACT PARTIAL SOLUTION TO THE STEADY-STATE, COMPRESSIBLE FLUID FLOW PROBLEMS OF JET FORMATION AND JET PENETRATION
}

\author{
by
}

\author{
Robert R. Karpp
}

\begin{abstract}
This report treats analytically the problem of the symmetric impact of two compressible fluid streams. We assume that the flow is steady, plane, inviscid, and subsonic and that the compressible fluid is of the Chaplygin (tangent gas) type. In the analysis, the governing equations are first transformed to the hodograph plane where an exact, closed-form solution is obtained by standard techniques. The distributions of fluid properties along the plane of symmetry as well as the shapes of the boundary streamlines are exactly determined by transforming the solution back to the physical plane. The problem of a compressible fluid jet penetrating into an infinite target of similar material is also exactly solved by considering a limiting case of this solution. This new compressible flow solution reduces to the classical result of incompressible flow theory when the sound speed of the fluid is allowed to approach infinity. Several illustrations of the differences between compressible and incompressible flows of the type considered are presented.
\end{abstract}

\section{INTRODUCTION}

The flow configuration involving the symmetric impact of plane jets or the impact of a single jet upon a rigid plane boundary has significant technological interest. For example, the extremely high velocity produced by jets from lined-cavity shaped charges has been explained by the steady-state model of impacting jets. ${ }^{1}$ Even the stretching of the jet has been explained by a variant of this model. ${ }^{2}$ This same model also explains some of the features associated with the bonding of metals in explosive welding. ${ }^{3}$

Several finite-difference computer codes exist that can be used to solve the type of problem discussed above. However, the accuracy of some of these codes sometimes is questioned, especially in regions of rapidly varying properties. The exact solution of this steady-state problem can be used to check the accuracy of approximate solutions obtained with hydrodynamic computer codes. In addition to developing a nontrivial test problem, another purpose of this work is to gain some understancing of how compressibility affects jet formation and jet penetration.

In this report, we first state the problem precisely. Then we put the equations governing this flow into a form suitable for solution and state the boundary conditions. After the compressible flow equations are specialized to a pressure-density relationship of the Chaplygin type, a well-known transformation is used to cast the governing equations in the form of Cauchy-Riemann equations with the velocity potential and 
stream function as dependent variables and the velocity components as independent variables. An exact solution for the complex stream function, as a function of the velocity components, is then obtained for the stated problem. However, transformation back to the physical plane has not been found possible for the entire flow field; only for two specific regions, for the shape of free surface streamlines and for all properties along the plane of symmetry, has the transformation been achieved. By taking a limiting case of the exact solution, we also obtain a partial solution to the problem of steady-state jet penetration. Plots of these solutions are presented for both the jet formation and jet penetration problems, and compressible and incompressible flows are compared in detail.

\section{STATEMENT OF THE PROBLEM}

The steady-state, symmetric impact of two fluid jets, in plane flow geometry, is considered. Figure 1 shows the configuration of this problem. The streams of incoming fluid are inclined to the $x$-axis, which is the plane of symmetry, at an angle $a$, where $0<a \leq \pi$. We assume that the fluid is inviscid, thermally nonconducting, and compressible. The widths of the streams asymptotically approach $h_{1}, h_{2}$, and $h_{3}$ away from the stagnation region. The incoming velocity, with magnitude $U_{e}$, is assumed to be uniform across the streams far from the stagnation region.

The pressure acting on the free streamlines, which are indicated by $\psi_{1}, \psi_{2}, \psi_{3}$, and $\psi_{4}$ in Fig. 1 , is taken to be zero. The pressure within the jets at infinity is also zero. When we apply Bernoulli's equation, we find that the magnitude of the velocity along all free streamlines is $U_{c}$. Applying the conservation equations of mass and momentum, we obtain the equations

$$
h_{2}=h_{1}(1+\cos \alpha)
$$

and

$$
h_{3}=h_{1}(1-\cos a) \text {, }
$$

which relate the widths of the outgoing streams to the widths of the incoming streams and the angle of incidence $\alpha$. Thus, the asymptotic conditions of the jets are completely specified, and the problem is to determine the details of the flow in the region of jet interaction.

\section{GOVERNING EQUATIONS}

The equations that are assumed to govern steady, plane flow are

$$
\begin{aligned}
& \frac{\partial}{\partial x}(p u)+\frac{\partial}{\partial y}(p v)=0, \\
& q d q+\frac{1}{\rho} d P=0, \\
& \frac{\partial u}{\partial y}-\frac{\partial v}{\partial x}=0,
\end{aligned}
$$

and

$$
P=P(p) \text {, }
$$

where $q=\left(u^{2}+v^{2}\right)^{1 / 2}$. In these equations, $u$ and $v$ are the $x$-and $y$-components of velocity, $q$ is the speed, $P$ is the pressure, and $\rho$ is the density. Equation (3) is a statement of the conservation of mass, and Eq. (4) is Bernoulli's equation. Equation (5) is the condition of irrotationality, and Eq. (6) is the pressure-density relation for the process. Equations (4) and (5) are used in place of the $x$-and $y$-components of the 
momentum equation. This simplifiction is justified for inviscid flow if an equation of the form of Eq. (6) exists and if the incoming flow is irrotational. Equations (3) through (6) are to be solved for the dependent variables $u, v, P$, and $\rho$.

Before we attempt a solution, we can cast the mathematical problem into a more standard form by introducing new dependent variables and by transforming to velocity components as independent variables. First, Eqs. (3), (4), and (6) can be combined to form

$$
\left(1-\frac{u^{2}}{c^{2}}\right) \frac{\partial u}{\partial x}-\frac{u v}{c^{2}}\left(\frac{\partial v}{\partial x}+\frac{\partial u}{\partial y}\right)+\left(1-\frac{v^{2}}{c^{2}}\right) \frac{\partial v}{\partial y}=0 \text {. }
$$

where

$$
c^{2} \equiv \frac{d P}{d p}
$$

is the sound speed squared. Equations (4) and (6) can be combined to produce a relation between the speed $q$ and the density $p$. This relation combined with the definition of sound speed, Eq. (8), could be used to produce a relationship of the form

$$
c=c(q) \text {. }
$$

The system of equations to be solved is

$$
\left(1-\frac{u^{2}}{c^{2}}\right) \frac{\partial u}{\partial x}-\frac{u v}{c^{2}}\left(\frac{\partial v}{\partial x}+\frac{\partial u}{\partial y}\right)+\left(1-\frac{v^{2}}{c^{2}}\right) \frac{\partial v}{\partial y}=0
$$

and

$$
\frac{\partial u}{\partial y}-\frac{\partial v}{\partial x}=0
$$

where the sound speed $c$ is a function of the particle speed $q=\left(u^{2}+v^{2}\right)^{1 / 2}$, as indicated by Eq. (9).

Equations (7) and (5) form a system of two equations for the two unknown functions $u$ and $v$. However, Eq. (7) is highly nonlinear. This nonlinearity can be removed by transforming to the hodograph variables $u$ and $v$ as independent variables. In addition, introducing a velocity potential $\phi$ and a stream function $\psi$ is also advantageous. These new dependent variables are defined by

$$
\frac{\partial \psi}{\partial x}=u, \quad \frac{\partial \psi}{\partial y}=v, \quad \frac{\partial \psi}{\partial x}=-\frac{p}{p_{0}} v \text {, and } \quad \frac{\partial \psi}{\partial y}=\frac{p}{p_{0}} u .
$$

Equations (10) can be combined with Eqs. (5) and (7) to give

$$
\frac{\rho}{\rho_{0}}\left(\frac{\partial \phi}{\partial u} v-\frac{\partial \phi}{\partial v} u\right)+\frac{\partial \psi}{\partial u} u+\frac{\partial \psi}{\partial v} v=0
$$

and

$$
e_{\rho_{0}}\left(\frac{\partial \psi}{\partial v} v+\frac{\partial \psi}{\partial u} u\right)+\left(1-\frac{u^{2}+v^{2}}{c^{2}}\right)\left(\frac{\partial \psi}{\partial v} u-\frac{\partial v}{\partial u} v\right)=0 \quad .
$$

which are the equations for $\phi$ and $\psi$ in the hodograph plane. The detailed derivation of Eqs. (11) and (12) is in Ref. 4. These equations take a simpler form when the polar coordinates $q$ and $\theta$ are used in place of $u$ and $v$. The variables $q$ and $\theta$ are defined by

$$
u=q \cos \theta \text {, and } \quad v=q \sin \theta \text {. }
$$

With Eqs. (13), Eqs. (11) and (12) become 


$$
\frac{E}{f_{0}} \frac{\partial \phi}{\partial \dot{E}}=q \frac{\partial \psi}{\partial q}
$$

and

$$
\frac{\rho}{\rho_{0}} q \frac{\partial \phi}{\partial q}=-\left(1-\frac{q^{2}}{c^{2}}\right) \frac{\partial \psi}{\partial \theta}
$$

From Eqs. (4) and (6), both $c$ and $\rho / \rho_{0}$ can be expressed as functions of the speed $q$ for any specific form of Eq. (6). Equations (14) and (15) are the two equations to be solved for $\phi(q, \theta)$ and $\psi(q, \theta)$.

Equations (14) and (15) simplify considerably when specialized to particular forms of the pressure-density relation, Eq. (6). ${ }^{3}$ The greatest simplification, of course, is the specialization to the case of an incompressible fluid, that is, when $p$ is a constant. For this case, $\rho / \rho_{o}=1, q / c=0$, and Eqs. (14) and (15) assume the simple forms

$$
\frac{\partial \phi}{\partial \theta}=q \frac{\partial \psi}{\partial q}
$$

and

$$
q \frac{\partial \phi}{\partial q}=-\frac{\partial \psi}{\partial \theta}
$$

Equations (16) and (17) are the Cauchy-Riemann equations, in polar coordinates (q, $-\theta$ ), for the conjugate harmonic functions $\phi$ and $\psi$. For the incompressible case, the stated problem reduces to a fairly standard problem in potential theory, and the exact solution to the complete problem has been obtained. ${ }^{6}$

For compressible flow, Eqs. (14) and (15) can be similarly simplified if the Chaplygin ${ }^{3}$ equation of state (or tangent gas) is used for Eq. (6). The Chaplygin equation is

$$
P=P_{1}-\frac{k^{2}}{p},
$$

where $P_{1}$ and $k$ are constants. When we use Eq. (18), we ran write Eqs. (14) and (15) as

$$
\frac{\partial \phi}{\partial \theta}=\left(1+\frac{q^{2}}{c_{0}^{2}}\right)^{1 / 2} q \frac{\partial \psi}{\partial q}
$$

and

$$
\left(1+\frac{q^{2}}{c_{o}^{2}}\right)^{1 / 2} q \frac{\partial \phi}{\partial q}=-\frac{\partial \psi}{\partial \theta},
$$

where $c_{0}$ is the sound speed at the stagnation point. Equations (19; and (20) can be simplified further by introducing a new independent variable $\mathrm{q}_{\mathrm{p}}$, defined by

$$
q_{1}=\frac{2 q}{1+\left(1+q^{2} / c_{0}^{2}\right)^{1 / 2}}
$$

Equations (19) and (20) become

$$
\frac{\partial \psi}{\partial \theta}=q_{1} \frac{\partial \psi}{\partial q_{1}}
$$

and

$$
q_{1} \frac{\partial \phi}{\partial q_{1}}=-\frac{\partial \psi}{\partial \theta} .
$$


Note the similarity between the system of Eqs. (22) and (23), which are the governing equations for the steady, plane flow of a compressible fluid with a pressure-density relation given by Eq. (18), and the system of Eqs. (16) and (17), which are the governing equations for incompressible flow. Because of this similarity, the speed variable $q_{i}$ plays the role of an incompressible speed. The jet formation problem is solved by obtaining a solution to Eqs. (22) and (23) that is consistent with appropriate boundary conditions.

\section{BOUNDARY CONDITIONS}

The strean function $\psi$, defined by Eqs. (10), has a constant value along streamlines. Also, the mass rate of flow per unit depth between two streamlines is proportional to the difference in the values of the stream function on those streamlines. The specific formula for the mass rate of flow per unit depth between two arbitrary streamlines is

$$
\dot{m}_{A-B}=p_{0}\left(\psi_{B}-\psi_{A}\right),
$$

where $\psi_{A}$ and $\psi_{B}$ are the values of the stream function along the two streamlines. Figure 2 shows the free streamlines in the hodograph plane. The value of the stream function is constant along each free streamline. The physical positions of these streamlines are indicated in Fig. I.

Because the stream function is determined only to within an arbitrary constant, the value of $\psi_{1}$ will be taken as zero. The remaining values of $\psi$ slong the free streamlines are determined from Eq. (24) with due account taken of the flow direction. The values of $\psi$ along the free streamlines are, therefore,

$$
\psi_{1}=0, \quad \psi_{2}=-D_{c} h_{1} \theta_{-} / D_{0}, \quad \psi_{3}=-D_{c}\left(h_{1}-h_{3}\right) 0_{-} / D_{0} .
$$

and

$$
\psi_{4}=-v_{c} h_{2} D_{-1} / 0_{0}
$$

These equations represent the boundary conditions for $\psi$.

\section{THE COMPLEX STREAM FUNCTION IN THE HODOGRAPH PLANE}

Because Eqs. (22) and (23) are the Cauchy-Riemann equations in the polar coordinates $\left(q_{1},-\theta\right)$ for the functions $\phi$ and $\psi$, these equacions guarantee that $\phi$ and $\psi$ can be combined into a single complex function $f$ of a single complex variable $\bar{w}$, as

$$
f(\pi)=\left(q_{1},-\theta\right)+1 \phi\left(q_{1},-\theta\right) \text {. }
$$

where $\bar{w}=q_{1} e^{-1 \theta}$. Any function of the form of Eq. (26) will satisfy the governing flow equations, Eqs. (22) and (23). The explicit form of the solution $f$ is specified by the boundary conditions, Eqs. (25), and can be computed from the formula of Schwarz. ${ }^{6}$ This formula expresses the value of a function $F(z)$ at a point 2 inside a circle of radius $R>|z|$ in terms of the values of its real part on the circle. If $F(z)=U+i V$, then we have

$$
F(z)=\frac{1}{2 \pi} \int_{0}^{2 \pi} U\left(R e^{i B}\right) \frac{R e^{i B}+z}{R e^{i \beta}-z} d B .
$$

where the contour of integration is the circle of radius $R$ centered at $z=0$. By applying this equation to our system with $\mathbf{F}(\mathbf{z})=-\mathrm{if}(\mathbf{z})$, we obtain 


$$
\begin{aligned}
& -1 f(\bar{v})=\psi-1 \phi \\
& =\frac{1}{2 \pi} \int_{0}^{2 \pi} \psi\left(U_{i} e^{i B}\right) \frac{U_{i} e^{i B}+\bar{y}}{U_{i} e^{i B}-\bar{v}} d B \\
& =\frac{1}{2 \pi}\left[\int_{0}^{a} \psi_{1} \frac{u_{i} e^{1 \theta}+\bar{w}}{u_{i} e^{1 \beta}-\bar{w}} d \theta+\int_{a}^{\pi} \psi_{2} \frac{u_{1} e^{1 \beta}+\bar{w}}{u_{i} e^{i \beta}-\bar{w}} d \theta\right. \\
& \left.+\int_{\pi}^{\pi-\alpha} v_{3} \frac{v_{1} e^{i_{B}}+\bar{w}}{v_{i} e^{i_{B}}-\bar{w}} d_{B}+\int_{2 \pi-\alpha}^{2 \pi} v_{4} \frac{v_{1} e^{i_{B}}+\bar{w}}{v_{1} e^{I_{B}}-\bar{w}} d \theta\right],
\end{aligned}
$$

where $U_{1}$ is the transformed maximum spe: $J$, that is,

$$
v_{1}=2 v_{c} /\left[1+\left(1+v_{c}^{2} / c_{0}^{2}\right)^{1 / 2}\right] \text {. }
$$

obtained from Eq. (21). The integrated form of Eq. (28) can be written, to within a constant, as

$$
\begin{aligned}
f(\bar{v})= & \frac{\rho_{0}}{D_{0}} \frac{v_{c}}{\pi}\left[\left(v_{1}-v_{2}\right) \ln \left(1-\frac{\bar{y}}{v_{1} e^{1 \alpha}}\right)\right. \\
& +\left(v_{3}-v_{4}\right) \ln \left(1-\frac{\bar{v}}{v_{1} e^{-1 \alpha}}\right) \\
& \left.+\left(v_{4}-v_{1}\right) \ln \left(1-\frac{\bar{v}}{v_{1}}\right)+\left(v_{2}-v_{3}\right) \ln \left(1+\frac{\bar{v}}{v_{1}}\right)\right] .
\end{aligned}
$$

If we use the boundary values of $\psi$ from Eqs. (25), we can put the complex stream function $f$ into the form

$$
\begin{aligned}
& f(\bar{v})=\frac{P_{0}}{D_{0}} \frac{U_{c}}{\pi}\left[h_{1} \ln \left(1-\frac{\bar{z}}{U_{1} e^{1 \alpha}}\right)\right. \\
& +n_{1} \ln \left(1-\frac{\bar{u}}{u_{1} e^{-1 a}}\right) \\
& \left.-a_{2} \ln \left(1-\frac{\bar{v}}{v_{l}}\right)-n_{3} \ln \left(1+\frac{\bar{v}}{v_{1}}\right)\right] \text {. }
\end{aligned}
$$

Equation (31) represents the exact solution to the stated problem in the hodograph plane. When this expression for $f$ is divided into real and imaginary parts, we obtain the velocity potential $\phi$ and the stream function $\psi$ that satisfy the governing equations, Eqs. (22) and (23). The stream function also satisfies the boundary conditions given by Eqs. (25).

\section{PARTIAL SOLUTION IN THE PHYSICAL PLANE}

We have obtained the exact solution in the hodograph plane; now we need to transform the solution back to the physical plane $(\mathrm{z}=\mathrm{x}+\mathrm{iy})$. To this end, consider the expression 


$$
\begin{aligned}
q e^{-1 \theta} d z & =(u-i v) d z \\
& =u d x+v d y-1(v d x-u d y) \\
& =\frac{\partial \psi}{\partial x} d x+\frac{\partial \varphi}{\partial y} d y-1\left(-\frac{P_{0}}{p} \frac{\partial \psi}{\partial x} d x-\frac{P_{0}}{p} \frac{\partial \psi}{\partial y} d y\right) \\
& =d \varphi+1 \frac{\rho_{0}}{p} d \psi
\end{aligned}
$$

If we restrict this discussion to a single, arbitrary streamline, then $d \psi=0$, and along that streamline

$$
d x=\frac{1}{q} e^{10} d \theta
$$

and $d f=d \phi$. Using Eqs. (31) and (21), we can write Eq. (33) as

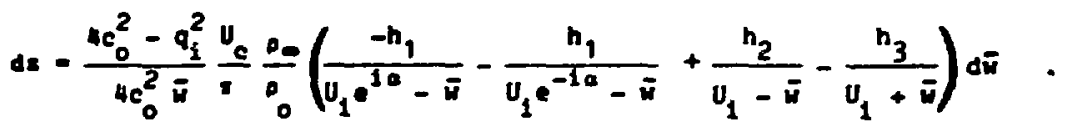

Because of the factor $q_{1}^{2}$, the right-hand side of the above expression is not a function solely of the complex variable $\bar{w}$. Therefore, to integrate Eq. (34), we must divide the expression on the right-hand side into its real and imaginary parts. Equation (34) can be written as

$$
d z=I\left(q_{1}, 0\right)\left(0^{-10} d q_{1}-1 a_{1} e^{-10} d \theta\right) \text {. }
$$

where $F\left(q_{1}, \theta\right)$ is the complex function preceding $d \bar{w}$ in Eq. (34). The relationship between $q_{1}$ and $\theta$, which is necessary to integrate Eq. (35), can be obtained, in principle, from Eq. (31). For any particular streamline, $\psi=$ constant $=\psi_{e}$, Eq. (31) furnishes a relation between $q_{1}$ and $\theta$, namely

$$
t_{c}=\text { In [f(E)]. }
$$

For a general streamline, it is difficult algebraically to solve Eq. (36) for $q_{1}$ as a function of $\theta$. At this point in the analysis, the problem has been reduced to one of solving an ordinary differential equation. This solution could be accomplished by numerical techniques. However, two particular cases, the case of free streamlines and the case of flow along the plane of symmetry, are treated exactly.

For free streamlines, $q_{1}=U_{1}$ (a constant), and $d q_{1}=0$. Equation (35) then becomes

$$
d z=F\left(v_{1}, \theta\right)\left(-1 u_{1} e^{-1 \theta} d \theta\right)
$$

or, when expanded,

$$
d z=-\frac{1}{1} \frac{D_{0}}{D_{0}}\left(-\frac{h_{1}}{e^{1 a}-e^{-1 \theta}}-\frac{h_{1}}{e^{-1 a}-e^{-1 \theta}}+\frac{h_{2}}{1-e^{-1 \theta}}-\frac{h_{3}}{1+e^{-1 \theta}}\right) d \theta .
$$

The solution to Eq. (38) is

$$
\begin{aligned}
z-z_{0} & =\frac{D_{0}}{T D_{0}}\left\{h_{2} e^{-1 \alpha} \ln \left[e^{1(\theta+a)}-1\right]+h_{1} e^{1 \alpha} \ln \left[e^{1(\theta-a)}-1\right]\right. \\
& \left.-h_{2} \ln \left[e^{1 \theta}-1\right]+h_{3} \ln \left[e^{1 \theta}+1\right]\right\} .
\end{aligned}
$$

Equation (39) gives the physical shape of the free surface streamlines in terms of the parameter $\theta$. For example, the streamline indicated by $\psi_{1}$ in Fig. 1 is described by letting $\theta$ vary from $-a^{+}$to $0^{-}$in Eq. 
(39). Similarly, the streamline $\psi_{2}$ is described by allowing $\theta$ to vary from $-a^{-}$to $-(\pi / 2)^{+}$. We can obtairs the constant of integration, $z_{0}$, in Fq. (39) by specifying that $y \rightarrow(1 / 2) h_{2}$ as $\theta \rightarrow 0^{-}$and that the distance between the centerline of the incoming stream and the free streamline, as indicated in Fig. 1, should approach $(1 / 2) h$, as $\theta \rightarrow-a^{+}$. The constant of integration that satisfies these conditions, for the free streamline $\psi_{1}$, is

$$
z_{0}=\frac{1}{2} h_{1} \csc a-\frac{P_{0}}{\pi p_{0}} b_{1}-\left[\frac{1}{2} h_{2}\left(1-\frac{P_{0}}{P_{0}}\right)+\frac{P_{0}}{P_{0}} a_{3}\right] \cot a+1 \frac{1}{2} h_{2}\left(1-\frac{P_{0}}{P_{0}}\right) \text {. }
$$

where

$$
\begin{aligned}
n_{1}= & \frac{\pi}{2} h_{1} \cos a+\frac{1}{2} h_{1} \sin a \ln \left[(\cos 2 a-1)^{2}+\sin ^{2} 2 a\right] \\
& +h_{1} \cos a \tan ^{-1}\left(\frac{-81 n 2 a}{\cos 2 a-1}\right)-h_{2} \tan ^{-1}\left(\frac{-\sin a}{\cos a-1}\right)+h_{3} \tan ^{-1}\left(\frac{-\sin a}{\cos a+1}\right)
\end{aligned}
$$

and

$$
\begin{aligned}
b_{1}= & \frac{\pi}{2} h_{1} \sin a+\frac{1}{2} h_{1} \cos a \ln \left[(\cos 2 a-1)^{2}+\cos ^{2} 2 a\right] \\
& -b_{1} \ln a \cos ^{-1}\left(\frac{-\sin 2 a}{2 a-1}\right)-\frac{1}{2} h_{2} \times\left[(\cos a-1)^{2}+\cos ^{2} a\right] \\
& +\frac{1}{2} b_{3} \ln \left[(\cos a+1)^{2}+1 n^{2} a\right] .
\end{aligned}
$$

In a similar manner, we can obtain the constant of integration for the streamline $\psi_{2}$ by specifying that $y$ $\rightarrow(1 / 2) h_{3}$ as $\theta \rightarrow-(\pi / 2)^{+}$and that the distance between the centerline of the incoming stream and the free streamline $\psi_{2}$ approaches $(1 / 2) h_{1}$ as $\theta \rightarrow-a^{-}$. For these conditions, the constant of integration for the free streamline $\Psi_{2}$ becomes

$$
2_{0}=-\frac{1}{2} h_{1} \csc a-\frac{\rho_{0}}{\rho_{0}} b_{2}-\left[\frac{1}{2} h_{3}\left(1-\frac{\rho_{-}}{\rho_{0}}\right)+\frac{\rho_{-}}{\rho_{0}} \cdot a_{2}\right] \cot a+1 \frac{1}{2} h_{3}\left(1-\frac{\rho_{-}}{\rho_{0}}\right) \text {. }
$$

where

$$
\begin{aligned}
a_{2}= & -\frac{\pi}{2} h_{1} \cos a+\frac{1}{2} h_{1} \sin a \operatorname{sa}\left[(\cos 2 a-1)^{2}+\sin ^{2} 2 a\right] \\
& +h_{1} \cos a \cos ^{-1}\left(\frac{-\sin 2 a}{\cos 2 a-1}\right)-h_{2} \tan ^{-1}\left(\frac{-\sin a}{\cos a-1}\right) \\
& +h_{3} \operatorname{can}^{-1}\left(\frac{-\sin a}{\cos a+1}\right)
\end{aligned}
$$

and

$$
\begin{aligned}
b_{2}= & -\frac{\pi}{2} h_{1} \sin \alpha+\frac{1}{2} h_{1} \cos a \ln \left[(\cos 2 a-1)^{2}+\sin ^{2} 2 a\right] \\
& -h_{1} \sin a \tan ^{-1}\left(\frac{-\ln 2 a}{\cos 2 a-1}\right) \\
& -\frac{1}{2} h_{2} \ln \left[(\cos a-1)^{2}+\sin ^{2} a\right] \\
& +\frac{1}{2} h_{3} \ln \left[(\cos a+1)^{2}+\sin ^{2} a\right] .
\end{aligned}
$$


Equation (39) together with the constants defined by Eqs. (40) and (41) specifies the shape of the free streamlines in the upper half plane Im $z>0$. We then can determine the free streamlines in the lower half plane by reflecting the solution in the upper half plane about the $x$-axis.

In the second case for which Eq. (35) can be integrated, flow along the plane of symmetry $y=0, E q$. (35) can be simplified because $\theta$ equals either 0 or $-\pi$ depending upon whether the point considered is to the right or left of the stagnation point. Consider first the streamline of symmetry to the right of the stagnation point. For this case, $\theta=0$ along the streamline and, therefore, $d \theta=0$. Equation (35) then becomes

$$
d z=F\left(q_{1}, 0\right) d q_{1}
$$

or

$$
d z=\frac{4 c_{0}^{2}-q_{1}^{2}}{4 c_{0}^{2} q_{1}} \frac{v_{c}}{\pi} \frac{D_{0}}{D_{0}}\left(-\frac{h_{1}}{J_{1} e^{1 a}-q_{1}}-\frac{h_{1}}{J_{1} e^{-1 a}-q_{1}}+\frac{h_{2}}{U_{1}-q_{1}}-\frac{h_{3}}{U_{1}+q_{1}}\right) d q_{1} \text {. }
$$

The integrated form of this equation is

$$
\begin{aligned}
z-z_{0}= & \frac{u_{c}}{v_{1}} \frac{\rho_{c}}{\pi p_{0}}\left[h_{1}\left(e^{-1 \alpha}-\frac{u_{1}^{2} e^{1 a}}{4 c_{0}^{2}}\right) \ln \left(e^{1 \alpha}-\frac{q_{1}}{v_{1}}\right)\right. \\
& +h_{1}\left(e^{1 a}-\frac{u_{1}^{2} e^{-1 a}}{4 c_{0}^{2}}\right) \ln \left(e^{-1 \alpha}-\frac{q_{1}}{u_{1}}\right) \\
& \left.-h_{2}\left(1-\frac{v_{1}^{2}}{4 c_{0}^{2}}\right) \ln \left(1-\frac{q_{1}}{v_{1}}\right)+h_{3}\left(1-\frac{v_{1}^{2}}{4 c_{0}^{2}}\right) \ln \left(1+\frac{q_{1}}{v_{1}}\right)\right] .
\end{aligned}
$$

Equation (43) links the position along the $x$-axis with the magnitude of velocity $q_{1}$. The constant of integration, $z_{0}=x_{0}+i y_{0}$, can be adjusted to position the stagnation point as desired. In this analysis, we have been unable to link the position of the speed distribution with the position of the free streamlines. If $y_{0}=0$, then the imaginary part of Eq. (43) vanishes, and that equation represents a relationship between $x$ and $q_{j}$. If desired, $x_{0}$ can be determined such that $x=0$ is the stagnation point $q_{1}=0$. Equation (21) then links $q$ and $q$.

Equation (43) applies only to flow along the $x$-axis in the positive direction. For the remaining portion of the streamline of symmetry where flow is in the negative $x$-direction, $\theta=-\pi$, and we can again integrate Eq. (35). The result of this integration is the same as Eq. (43) except that $q_{1}$ is replaced by $-q_{1}$ and the right-hand side of Eq. (43) is preceded by a minus sign.

Once the velocity distribution along the plane of symmetry is determined, the remaining quantities of interest can be determined from the pressure-density relation, Eq. (18), and Bernoulli's equation, Eq. (4). The relevant relationships are

$$
\begin{array}{ll}
c^{2}-k^{2} / p^{2}, & c_{0}^{2}-c_{1}^{2}-q^{2}, \\
p^{2}-k^{2} / c^{2}, & \quad c^{2}-c_{0}^{2}+q^{2},
\end{array}
$$

and

$$
E=E_{-}-P_{1}\left(1 / 0-1 / 0_{0}\right)+1 / 2 k^{2}\left(1 / 0^{2}-1 / 0_{-}^{2}\right) .
$$

where $\mathrm{E}$ is the specific internal energy. The last relationship is determined from the energy equation dE =

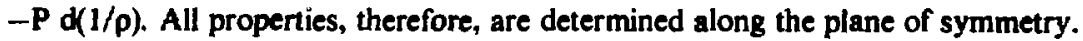


Summarizing this section, we find that the shape of the free surface streamlines is given by Eq. (39), with constants of integration prescribed by Eq. (40) or Eq. (41). The velocity distribution along the plane of symmetry is given by Eq. (43). Once the velocity distribution is determined, Eqs. (44) can be used to obtain the distribution of all other relevant quantities.

\section{PAKTIAL SOLUTION TO THE JET PENETRATION PROBLEM}

The foregoing solution can be specialized to the problem of the steady-state penetration of a jet into an infinite target by taking a limiting case of that solution. This limiting case is achieved by first allowing the angle of inclination $\alpha$ to approach zero while holding the jet thickness $h_{3}$ constant and then reversing the direction of flow. To be consistent, the thicknesses $h_{1}$ and $h_{2}$ must approach infinity. Figure 3 shows the resulting flow configuration. For incompressible flow, this transition from the jet formation problem to the jet penetration problem has been discussed by Birkhoff. ${ }^{3}$

In this limit, that is, $a \rightarrow 0$ with $h_{3}=$ constant, the expression for the free streamlines, Eq. (39), becomes

$$
z=z_{0}+\frac{\rho_{\Delta h_{3}}}{\rho_{0} \pi}\left[\frac{2\left(2-e^{-1 \theta}\right)}{\left(1-e^{-1 \theta}\right)^{2}}+20 \frac{e^{1 \theta}+1}{e^{1 \theta}-1}\right] .
$$

To satisfy the condition that $y \rightarrow(1 / 2) h_{3}$ as $\theta \rightarrow-\pi$, we must take

$$
y_{0}=\frac{1}{2} h_{3}\left(1-\frac{D_{m}}{P_{0}}\right)
$$

The constant $x_{0}$ in $z_{0}$ merely sets the position, in the horizontal direction, of the stagnation point. The distribution of velocity along the plane of symmetry is obtained from Eq. (43) by the above limiting process. The resulting equation is

$$
x-x_{0}-\frac{P_{\omega h} U_{c}}{P_{0} \pi U_{1}}\left\{\left(1-\frac{u_{1}^{2}}{4 c_{0}^{2}}\right)\left[\operatorname{lo} \frac{u_{1}+q_{1}}{U_{1}-q_{i}}+\frac{2 q_{i}}{u_{1}\left(1-q_{i} / u_{i}\right) 2}\right]+\frac{4\left(1+u_{i}^{2} / 4 c_{0}^{2}\right)}{1-q_{i} / u_{i}}\right\} \text {, }
$$

for flow in the positive $x$-direction. For flow in the negative $x$-direction, an equation similar to Eq. (47) holds with $q_{1}$ replaced by $-q_{1}$ and a minus sign preceding the right-hand side of Eq. (47). As before, we have established no link between the position coordinates in Eqs. (45) and (47). As in the previous section, the distribution of other relevant properties along the plane of symmetry is determined by Eqs. (44), once the velocity $q_{j}$, and therefore $q$, has been determined.

\section{RESULTS AND CONCLUSION}

The shapes of free surface streamlines are illustrated in Figs. 3-5. Figure 3 shows streamlines for jet penetration evaluated from Eq. (45). The streamlines shown in Figs. 4 and 5 were calculated from Eq. (39); they represent jet formation at impact angles of $90^{\circ}$ and $45^{\circ}$, respectively. The parameters used for all calculations of compressible flow in this work are $\mathrm{k}^{2}=12.18 \times 10^{5} \mathrm{GPa} \cdot \mathrm{kg} / \mathrm{m}^{3}, \rho_{\infty}=8.9 \times 10^{3}$ $\mathrm{kg} / \mathrm{m}^{3}, \mathrm{c}_{\infty}=3.92 \mathrm{~km} / \mathrm{s}, P_{1}=\mathrm{k}^{2} / \rho_{\infty}, U_{c}=3.53 \mathrm{~km} / \mathrm{s}\left(M_{\infty}=U_{c} / c_{\infty}=0.9\right)$. These values are appropriate for copper at normal initial conditions; however, they are not representative of copper at high pressures because the Chaplygin equation is a linear relation between $\mathbf{P}$ and $1 / p$. Therefore, these calculations generally overestimate the amount of compression that would occur in copper. We use $h_{1}=10.0 \mathrm{~mm}$ for Figs. 4 and 5 and $2 h_{3}=10.0 \mathrm{~mm}$ for Fig. 3. We also can obtain incompressible flow from these equations by assuming that $\mathrm{k}^{2} \rightarrow \infty$. In Figs. 3-5, the shapes of free surface streamlines for compressible flow (M⿻ 
$=0.9)$ and incompressible flow $\left(\mathrm{M}_{\infty}=0.0\right)$ are compared. From this work we conclude that the curvature of the compressible flow streamlines is greater than the curvature of incompressible streamlines, in regions of high curvature. This effect is evident directly from the factor $\rho_{\infty} / \rho_{0}$ in Eqs. (39) and (45). This factor is unity for incompressible flow and less than unity for compressible flow. Thus, the geometry of the streamlines is scaled down for compressible flow, and therefore, curves which are mild for incompressible flow become sharp for compressible flow.

Figures 6-15 show the distribution of properties along the plane of symmetry for the flow configurations illustrated in Figs. 3-5. Figures 6-10 give the property distributions of pressure, velocity, strain rate in the $x$-direction $(\partial u / \partial x)$, specific internal energy, and density for compressible and incompressibla flows at normal incidence (Fig. 4 shows the streamlines). These values are calculated from Eqs. (43) and (44). In addition to the obvious large difference in the distributions of density and internal energy between compressible and incompressible flows, there appears to be a significant difference in the strain-rate distribution. As shown in Fig. 8, the maximum values of strain rate occur some distance from the point of symmetry for compressible flow. For incompressible flow, the maximum is at the point of symmetry. The strain rate for the compressible flow is about $50 \%$ higher than that for incompressibie flow at the velocities considered. The pressure distribution also follows this trend. In Fig. 6, the region of significant pressure is confined to a smaller interval along the $\mathrm{x}$-axis for a compressible flow than for an incompressible flow. The velocity and other properties also follow this trend.

Figures 11 and 12 show the pressure and strain-rate distributions along the plane of symmetry for a flow with an incidence angle of $45^{\circ}$ (Fig. 5 shows the streamlines). The strain-rate distribution in Fig. 12 indicates that the compressible flow has a maximum value about twice as great as the incompressible flow maximum, and that the two distributions are qualitatively different.

Figures 13-15 show the pressure, velocity, and strain-rate distributions along the plane of symmetry for trie jet penetration problem illastrated in Fig. 3. The differences in distributions between compressible and incompressible flows are similar to those for the jet impact configurations.

Summarizing the results, we find that the curvature of free surface streamlines is greater for compressible flow than for incompressible flow. The pressure and strain-rate distributions along the plane of symmetry are greater for compressible flow than for incompressible flow. And, the strain-rate distribution is qualitatively different for compressible flow.

\section{REFERENCES}

1. G. Birkhoff, D. P. MacDougall, E. M. Pugh, and G. I. Taylor, "Explosives with Lined Cavities," J. Appl. Phys. 19, S63-582 (1948).

2. E. M. Pugh, R. J. Eichelberger, and N. Rostoker, "Theory of Jet Formation by Charges with Lined Conical Cavities," J. Appl. Phys. 23, 532-536 (1952).

3. G. R. Cowan and A. H. Holtzman, "Flow Configurations in Colliding Plates: Explosive Bonding," J. Appl. Phys. 34, 928-939 (1963).

4. R. Courant and K. O. Friedrichs, Supersonic Flow and Shock Waves (Interscience Publishers, Inc., New York, 1948), pp. 247-252.

5. G. Birkhoff and E. H. Zarantonello; Jets, Wakes, and Cavities (Academic Press, Inc., New York, 1957), pp. 185-189.

6. L. M. Milne-Thomson, Theoretical Hydrodynamics, 2nd Ed. (The MacMillan Co., New York, 1950), pp. 273-280. 


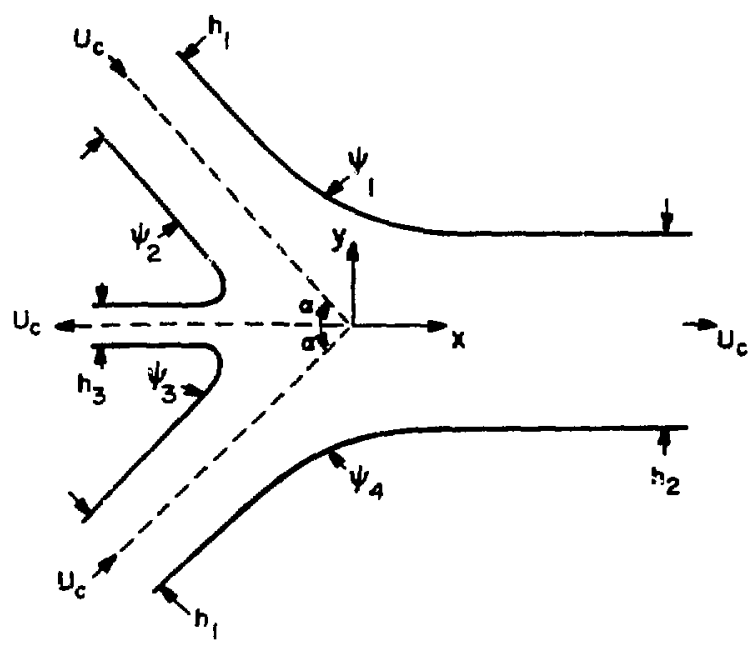

Fig. 1.

Physical plane indicating the symmetric impact of plane jets. The incoming velocity is $\mathbf{U}_{\mathbf{c}^{\cdot}}$

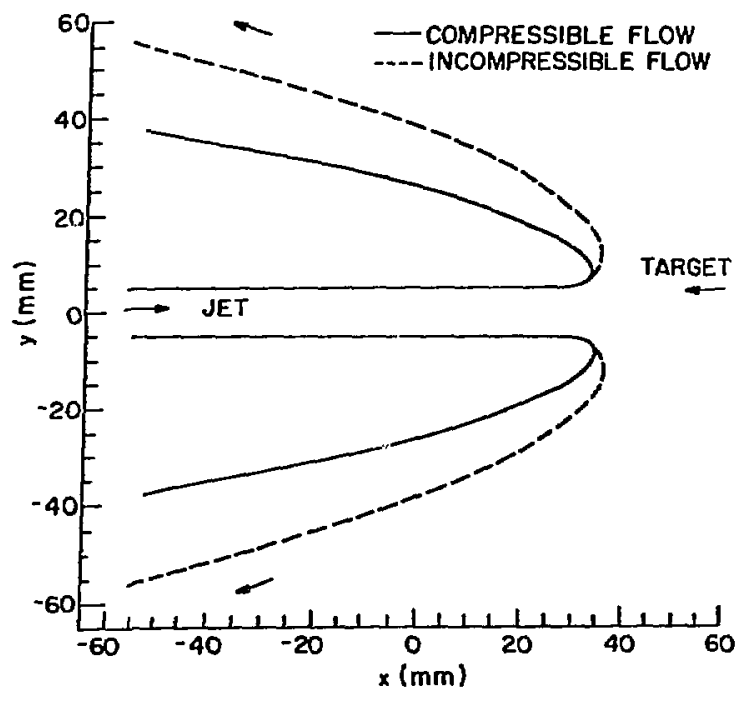

Fig. 3.

Streamlines for compressible and incompressible flows for jet penetration $\left(\alpha \rightarrow 0^{\circ}\right)$.

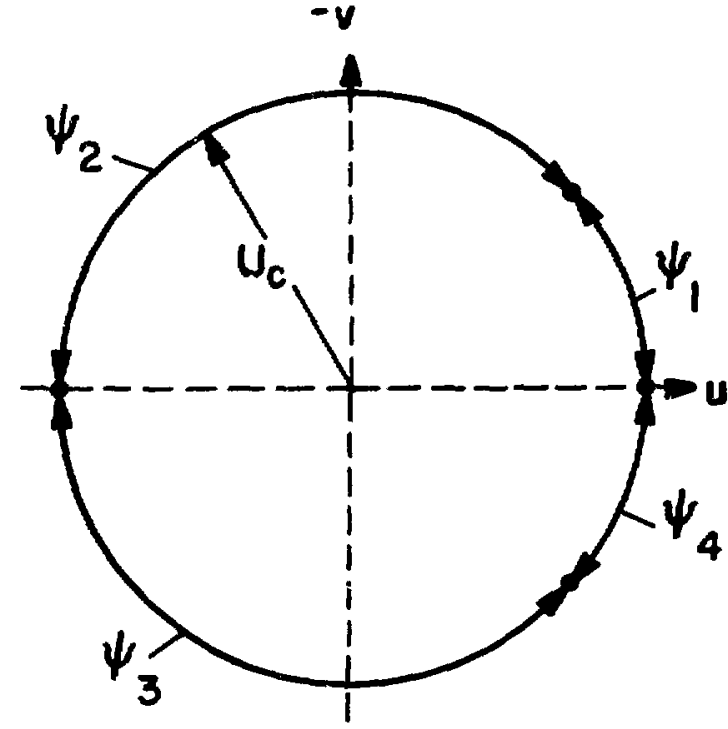

Fig. 2.

Hodograph plane indicating the constant values of the stream function along free surface streamlines.

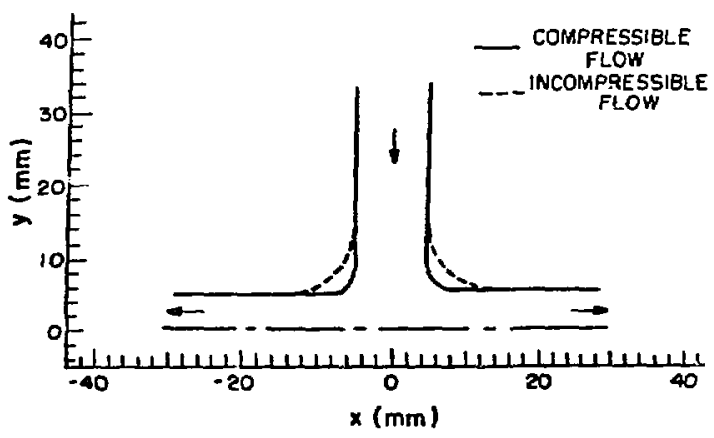

Fig. 4.

Streamlines for compressible and incompressible flows at normal incidence $\left(\alpha=90^{\circ}\right)$.

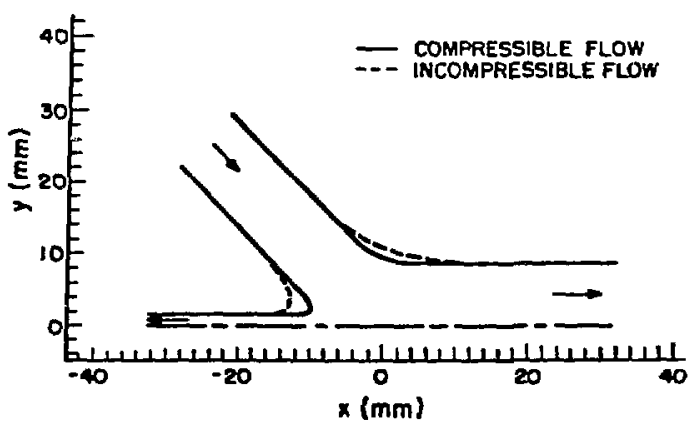

Fig. 5.

Streamlises for compressible and incompressible flows at an impact angle of $45^{\circ}$. 


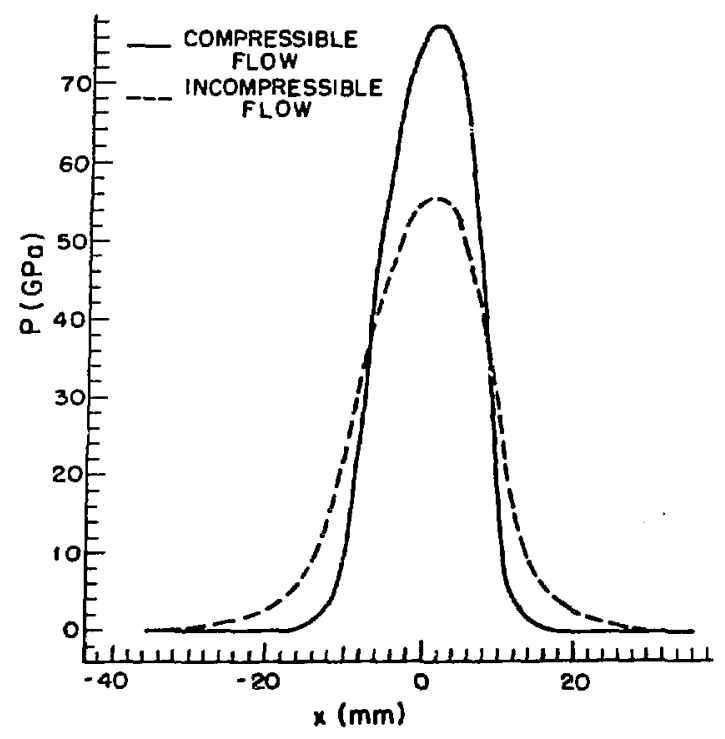

Fig. 6.

Pressure distribution along the plane of symmetry for normal incidence (streamlines shown in Fig. 4).

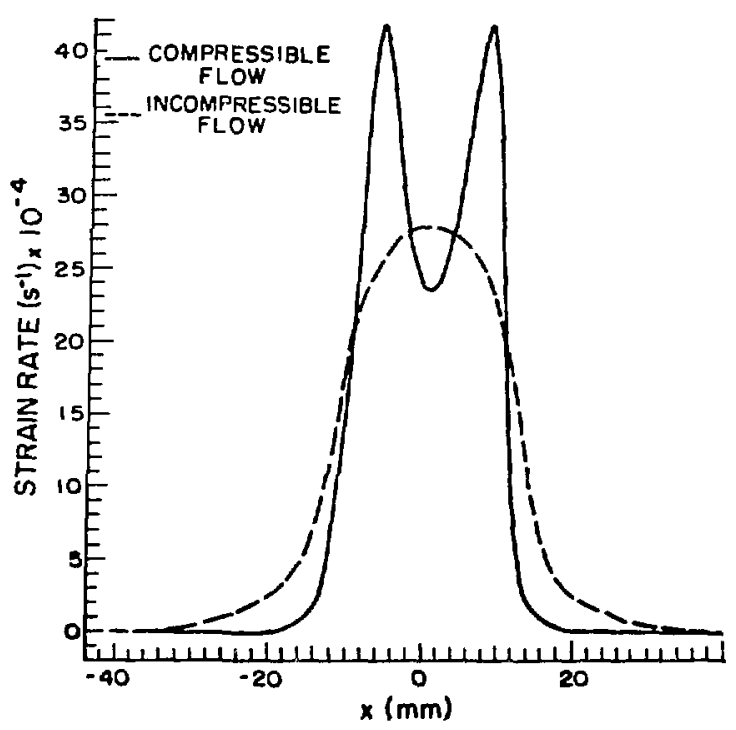

Fig. 8.

Strain-rate distribution along the plane of symmetry for normal incidence (streamlines shown in Fig. 4).

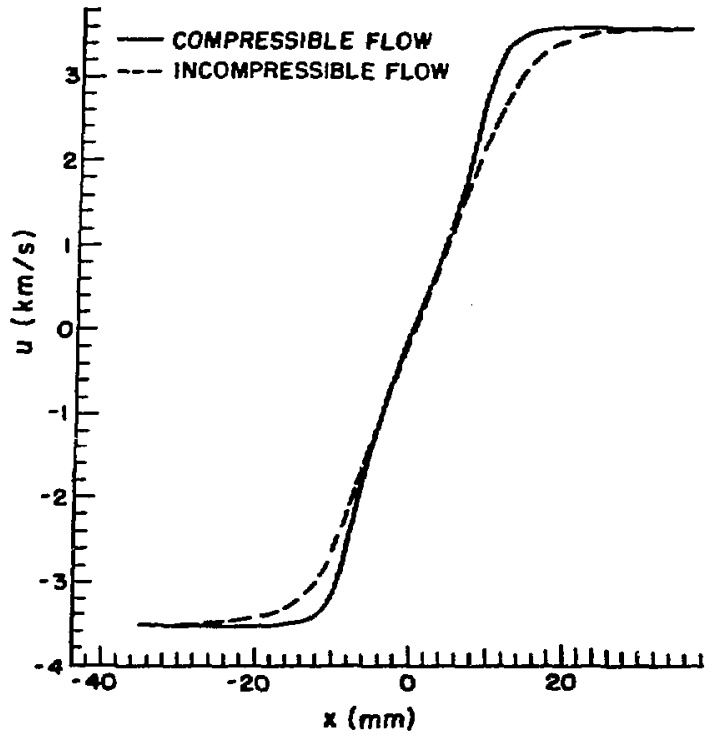

Fig. 7.

Velocity distribution along the plane of symmetry for rormul incidence (streamlines shown in Fig. 4).

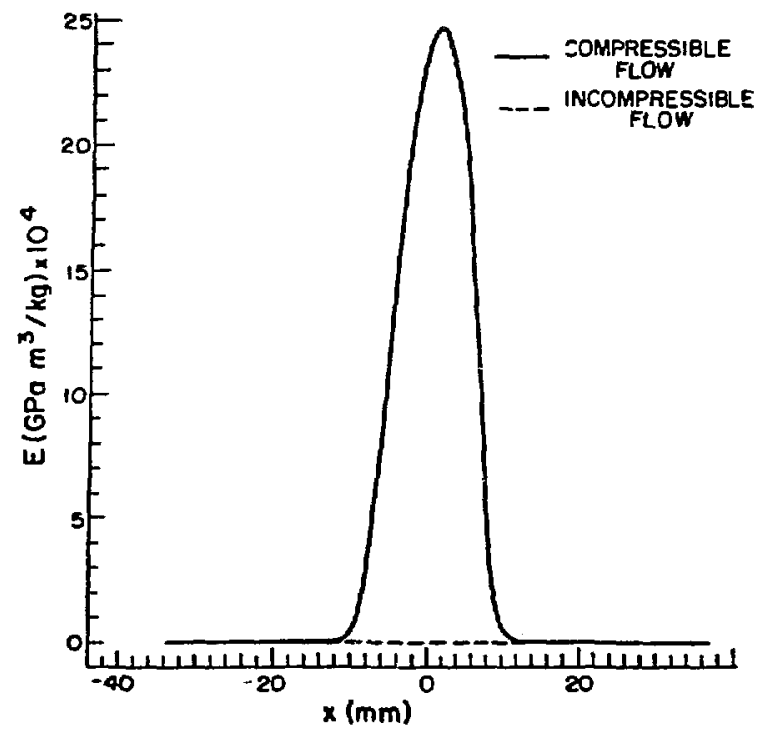

Fig. 9.

Specific internal energy distribution along the plene of $3 y m$ metry for normal ineidence (streamlines shown in Fig. 4). 


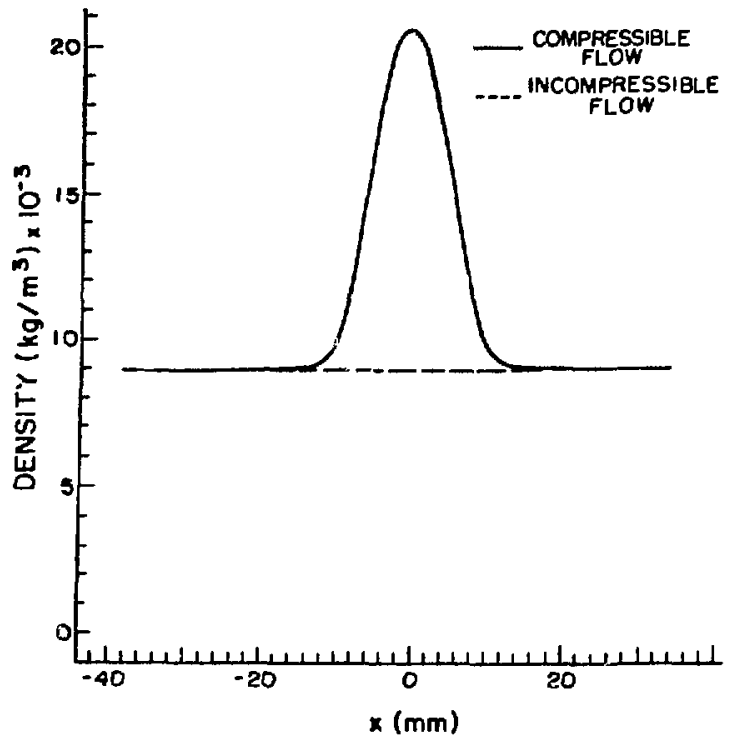

Fig. 10.

Density distribution along the plane of symmetry for normal incidence (streamlines shown in Fig. 4).

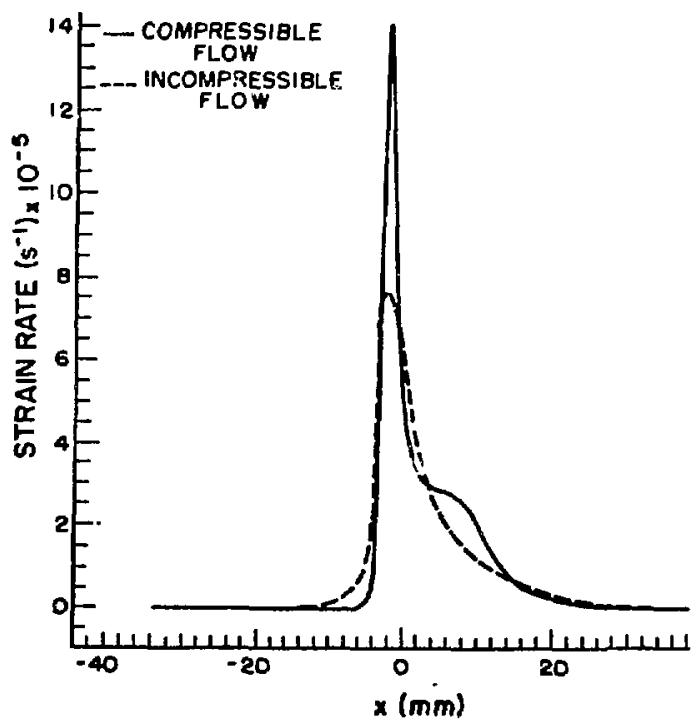

Fig. 12.

Strain-rate distribution along the plane of symmetry for an impact angle $a=45^{\circ}$ (streamlines shown in Fig. 5).

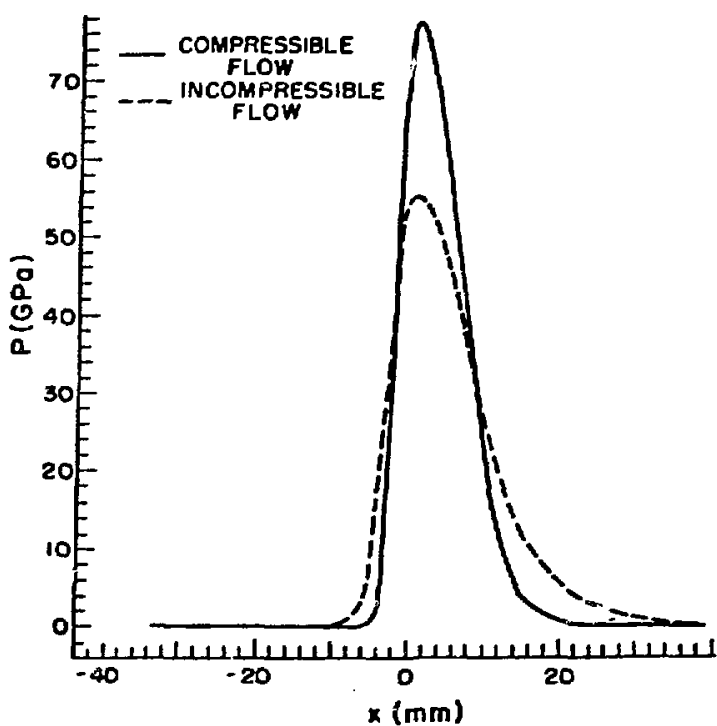

Fig. I1.

Pressure distribution along the plane of symmetry for an impact angle $a=45^{\circ}$ (streamlines shown in Fig. 5).

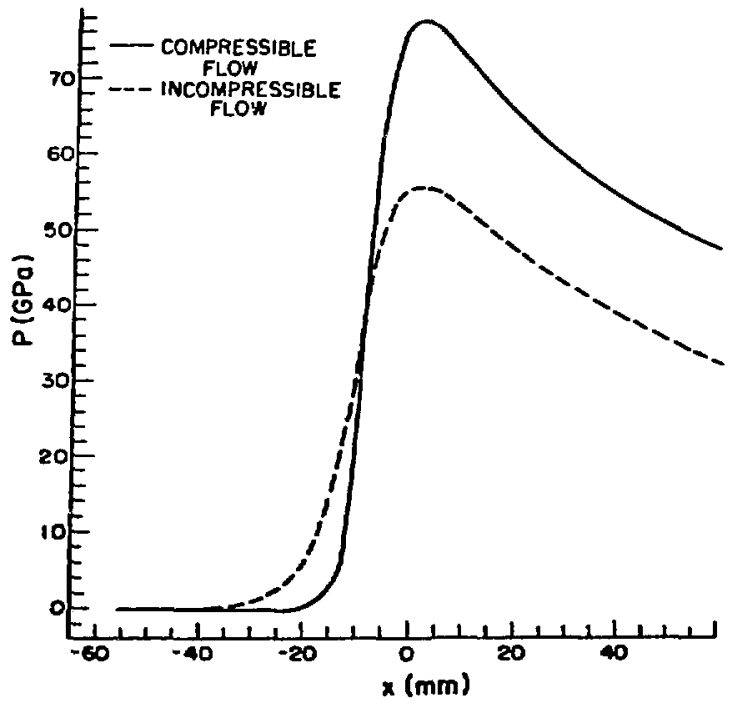

Fig. 13.

Pressure distribution along the plane of symmetry for jet penetralion (streamlines shown in Fig. 3). 


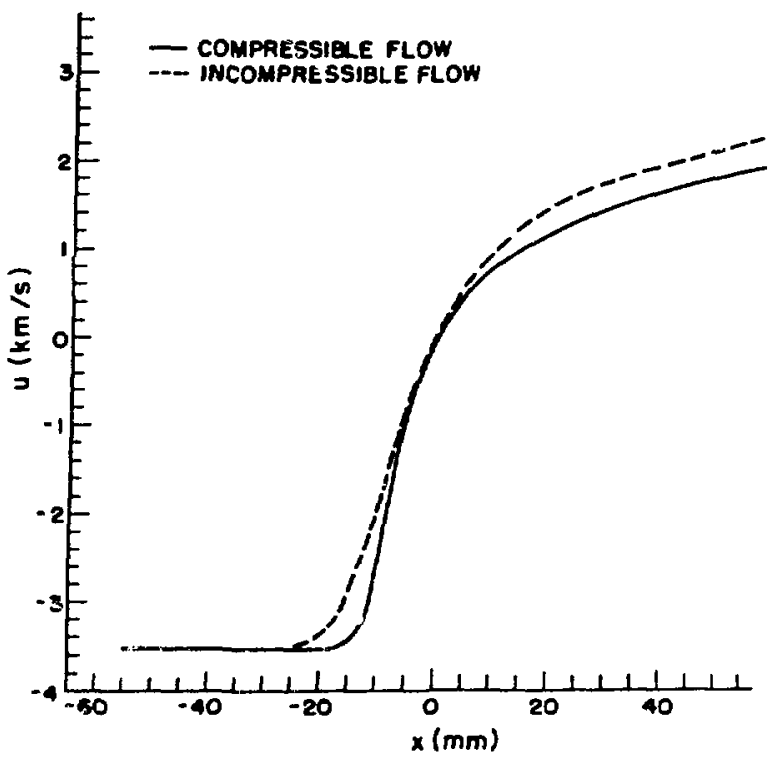

Fig. 14.

Velocity distribution slong the plane of symmetry for jet penetration (streamlines shown in Fig. 3).

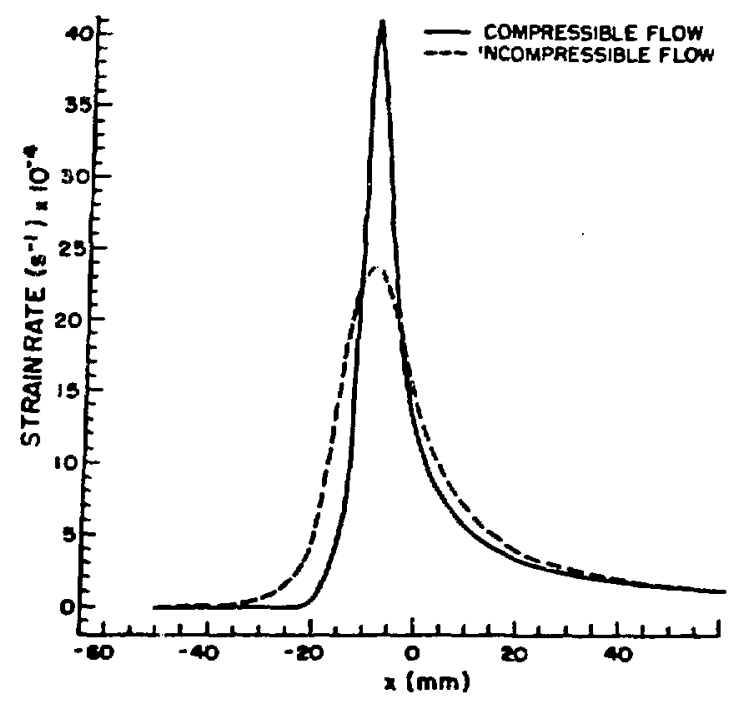

Fig. 15.

Strain-rate distribution along the plane of symmetry for jet penetration (streamlines shcwn in Fig. 3). 